\title{
Brachial Plexus Injuries
}

National Institute of Neurological Disorders and Stroke (NINDS)

\section{Source}

National Institute of Neurological Disorders and Stroke (NINDS). Brachial Plexus Injuries Information Page.

The brachial plexus is a network of nerves that conducts signals from the spine to the shoulder, arm, and hand. Brachial plexus injuries are caused by damage to those nerves. Symptoms may include a limp or paralyzed arm; lack of muscle control in the arm, hand, or wrist; and a lack of feeling or sensation in the arm or hand. Brachial plexus injuries can occur as a result of shoulder trauma, tumors, or inflammation. There is a rare syndrome called Parsonage-T urner Syndrome, or brachial plexitis, which causes inflammation of the brachial plexus without any obvious shoulder injury. This syndrome can begin with severe shoulder or arm pain followed by weakness and numbness. In infants, brachial plexus injuries may happen during birth if the baby's shoulder is stretched during passage in the birth canal.

The severity of a brachial plexus injury is determined by the type of damage done to the nerves. The most severe type, avulsion, is caused when the nerve root is severed or cut from the spinal cord. There is also an incomplete form of avulsion in which part of the nerve is damaged and which leaves some opportunity for the nerve to slowly recover function. Neuropraxia, or stretch injury, is the mildest type of injury. Neuropraxia damages the protective covering of the nerve, which causes problems with nerve signal conduction, but does not always damage the nerve underneath. 